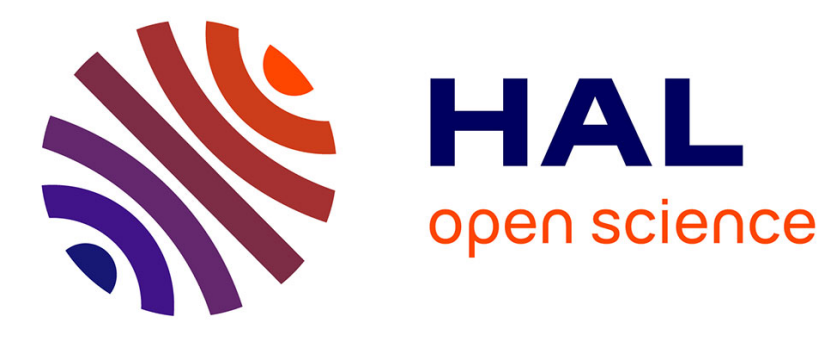

\title{
An Analytic Solution for the Perspective 4-Point Problem
}

Radu Horaud, Bernard Conio, Olivier Leboulleux, Bernard Lacolle

\section{To cite this version:}

Radu Horaud, Bernard Conio, Olivier Leboulleux, Bernard Lacolle. An Analytic Solution for the Perspective 4-Point Problem. Proceedings Computer Vision and Pattern Recognition, Jun 1989, SanDiego, United States. pp.500-507, 10.1109/CVPR.1989.37893 . inria-00589992

\section{HAL Id: inria-00589992 https://hal.inria.fr/inria-00589992}

Submitted on 16 Jun 2011

HAL is a multi-disciplinary open access archive for the deposit and dissemination of scientific research documents, whether they are published or not. The documents may come from teaching and research institutions in France or abroad, or from public or private research centers.
L'archive ouverte pluridisciplinaire HAL, est destinée au dépôt et à la diffusion de documents scientifiques de niveau recherche, publiés ou non, émanant des établissements d'enseignement et de recherche français ou étrangers, des laboratoires publics ou privés. 


\title{
An Analytic Solution for the Perspective 4-Point Problem
}

\author{
Radu Horaud, Bernard Conio, and Olivier Leboulleux, LIFIA \\ Bernard Lacolle, TIM3 \\ LIFIA-IMAG, 46 Avenue Félix Viallet, 38031 Grenoble \\ TIM3-IMAG, BP 68, 38402 Saint-Martin d'Hères \\ FRANCE
}

\begin{abstract}
The perspective n-point ( $\mathrm{PnP}$ ) problem is the problem of finding the position and orientation of a camera with respect to a scene object from $n$ correspondence points. In this paper we propose an analytic solution for the perspective 4-point problem. The solution is found by replacing the four points with a pencil of three lines and by exploring the geometric constraints available with the perspective camera model. We show how the P4P problem is cast into the problem of solving a biquadratic polynomial equation in one unknown. Although developped as part of an object recognition from a single view system [6], the solution might well be used for hand-eye coordination, landmark-guided navigation, and for fast determination of exterior camera parameters in general.
\end{abstract}

Key words: exterior camera calibration, 3D object recognition, location determination, visual navigation.

\section{Introduction}

One of the fundamental goals of Computer Vision is to discover properties that are intrinsic to a scene by analysing one or several images of this scene. Within this paradigm, an essential process is the determination of the position and orientation of the sensing device (the camera) with respect to objects in the scene. This problem is known as the exterior camera calibration problem and it has many interesting applications in Robotics and Cartography. Important applications in Robotics are: sensor calibration [13], object recognition and localisation from a single view [6], [9], [2], stereo sensor calibration [3], hand/eye coordination [14], and sensory based navigation [1].

In Cartography the problem is to determine the loca- tion in space from which an image or a photograph was obtained by recognizing a set of landmarks appearing in the image [4].

More formally, the problem may be stated as follows: Given a set of points with their coordinates in an object-centered frame and their corresponding projections onto an image plane and given the intrinsic camera paremeters, find the transformation matrix (three rotations and three translations) between the object frame and the camera frame (see Figure 1).

This problem is refered to as the perspective n-point problem and is usually solved using least-squares techniques. An elegant least-squares solution has recently been proposed [9]. Least-squares techniques require the computation of numerical solutions. For these solutions to be stable a large set of data points are needed which inherently augments the complexity of the computation. This is not desirable especially when the computation resides in the inner loop of a recognition/localisation process [6], [9], [5], [12], [7], [8], [2].

For these reasons a certain number of researchers have tried to determine the minimum number of points necessary to find a solution, and associated with each set of points they have tried to find a closed form expression of the problem. A finite number of solutions is available only when the number of points is equal or greater than 3 . The following is a brief review of the suggested solutions.

- Three points. Rives et al. [10] derive a set of three quadratic equations with three unknowns. These unknowns are the distances from the optical center of the camera to the three points. In theory there are 8 solutions. Fischler and Bolles [4] notice that for every real positive solution there is a real negative solution and hence a maximum of 4 solutions are in fact possible. They derive a closed form expression, namely a biquadratic polynomial 
equation in one unknown.

- Four points. When the points are coplanar a set of solutions can be found by considering any three among the four points and verifying the solution with the fourth point: A unique solution is thus found in [4]. When the points are not coplanar a closed form expression does not appear to have been derived. Rives et al. [10] solve a set of six quadratic equations with four unknowns. Fischler and Bolles [4] attack the problem by finding solutions associated with subsets of three points and selecting the solutions that they have in common. They provide a geometric construction which shows that unlike the coplanar case, two solutions may be available.

- Five points. For five points in general position the strategy mentioned above can as well be applied: Compare the solutions obtained with subsets of three or four points.

- Six points or more will always produce a unique solution: For six points we obtain 12 linear equations which are enough to determine the 12 coefficients of the transformation matrix, 9 for the rotations and 3 for the translations.

- Three lines. An alternative solution is to use lines instead of points. Horaud [6] suggests a constructive method for the case of a pencil of three non coplanar lines. Dhome et al. [2] solve for the general case of three arbitrary lines. The solutions are given by the roots of a polynomial of order 8 in one unknown.

In this paper we derive an analytic solution for the case of four non coplanar points, namely a biquadratic polynomial in one unknown. Roots of such an equation can be found in closed form or by an iterative method. Finding a solution for four non coplanar points is equivalent to finding a solution for a pencil of three non coplanar lines: The three lines share one of the four points. Notice that these lines may or may not correspond to physical linear edges in the scene. Figure 2 (a to d) shows various line and point configurations which are amenable to solving the P4P problem. The shaded lines are not physical edges. The configurations shown in Figure 2 ( $e$ and $f$ ) lead to more complex solutions [2], [11].

Finding a closed form solution for four non coplanar points is important for several reasons. First, they provide fewer solutions than three points. Second, the solutions are more stable when the points are not coplanar, because they do not depend of the relative orientation of the image plane with respect to the scene plane

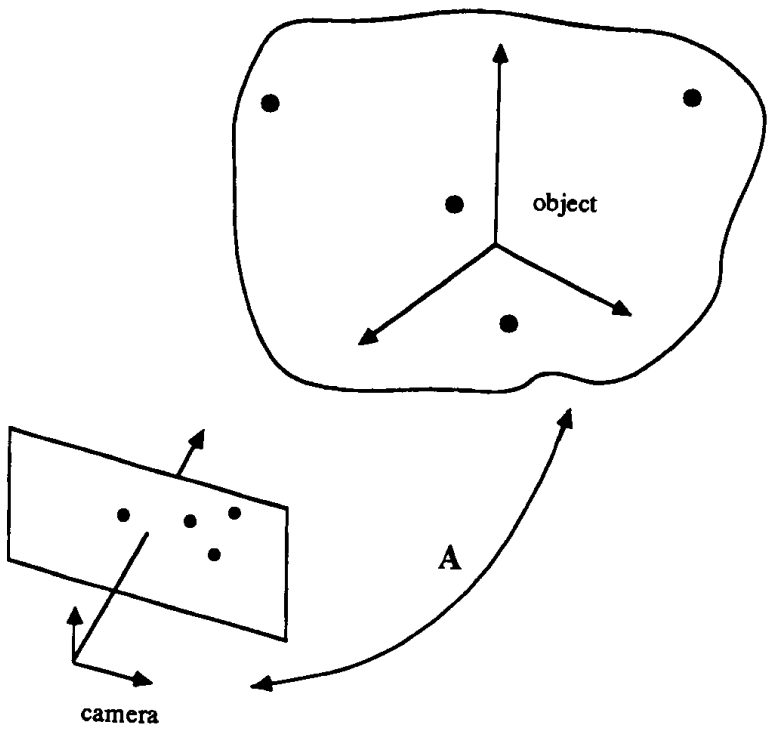

Figure 1: The transformation matrix between an object-centered frame and a camera-centered frame.

containing the points. Third, the computation of such a solution is very fast and therefore it can be included in a runtime visual process.

\section{The solution}

In order to compute the transformation matrix $\mathbf{A}$ of Figure 1 we decompose it into two matrices, $\mathbf{A}_{1}$ and $\mathbf{A}_{2}$, and we define three frames: a camera centered frame, an image centered frame, and an object centered frame, e.g., Figure 3. $\mathbf{A}_{1}$ is the transformation matrix from the image frame to the camera frame and $\mathbf{A}_{2}$ is the transformation matrix from the object frame to the image frame. Therefore we have:

$$
\mathbf{A}=\mathbf{A}_{1} \mathbf{A}_{2}
$$

The four non coplanar points, $M, M_{1}, M_{2}, M_{3}$ are replaced by a pencil of three non coplanar line segments, as shown on Figure 5 . We denote by $L_{1}, L_{2}$, and $L_{3}$ the unit vectors associated with the directions of these lines and let $l_{1}, l_{2}$, and $l_{3}$ be the unit vectors associated with their projections onto the image plane. The three frames used throughout the paper are defined as follows:

- The object coordinate system is defined as follows (see Figure 4). $L_{3}$ is the $\mathrm{x}$-axis. Let $P_{3}$ be a unit vector in the plane perpendicular to $L_{3}$. The geometric meaning of $P_{3}$ will be soon made clear. Let 


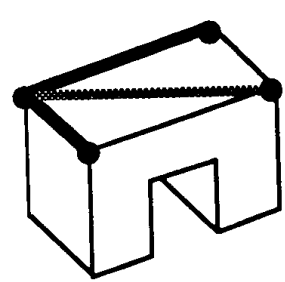

a) 4 coplanar points

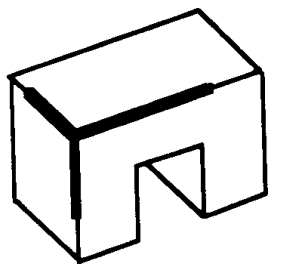

c) 3-line vertex

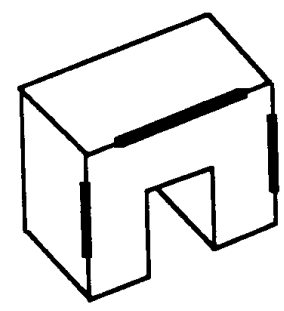

e) 3 coplanar lines

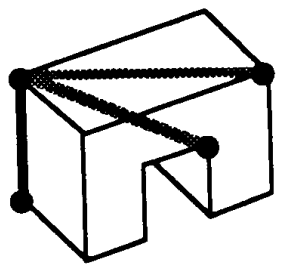

b) 4 non coplanar points

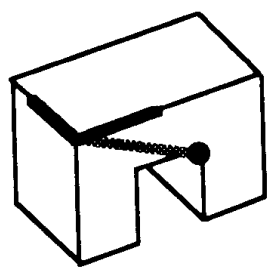

d) 2 lines and 1 point

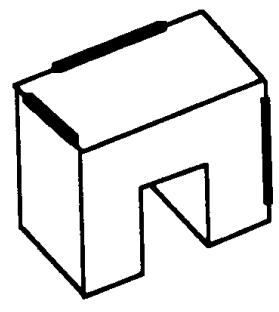

f) 3 non coplanar lines

Figure 2: Various point and line configurations on a 3-D object. The solution proposed in this paper can be applied to configurations $a, b, c$, and $d$.

$P_{3}$ be the $y$-axis of the object frame; the $z$-axis is defined by the cross product $L_{3} \times P_{3}$. It is easy to determine the object-frame coordinates of $L_{1}$ and $L_{2}$, e.g., Figure 4:

$$
\begin{array}{r}
L_{1}=\sin \alpha_{1} L_{3}+\cos \alpha_{1} \cos (\beta+\theta) P_{3}+ \\
\cos \alpha_{1} \sin (\beta+\theta) L_{3} \times P_{3} \\
L_{2}=\sin \alpha_{2} L_{3}+\cos \alpha_{2} \cos (\beta-\theta) P_{3}+ \\
\cos \alpha_{2} \sin (\beta-\theta) L_{3} \times P_{3}
\end{array}
$$

In these formulas $\alpha_{1}$ is the value of the angle between $L_{1}$ and $L_{1}^{\prime}$ (the projection of $L_{1}$ onto the yz-plane). The value of the angle between $L_{1}^{\prime}$ and $L_{2}^{\prime}$ is $2 \beta$. Notice that in these formulas $\theta$ is an unknown which will be determined.

- The camera coordinate system has its origin at the focal point $F$. The $z$-axis is the optical axis of the camera and the $x y$-plane is parallel to the image plane. The image is at distance $f$ (the focal length) from the origin along the $\mathrm{z}$-axis.

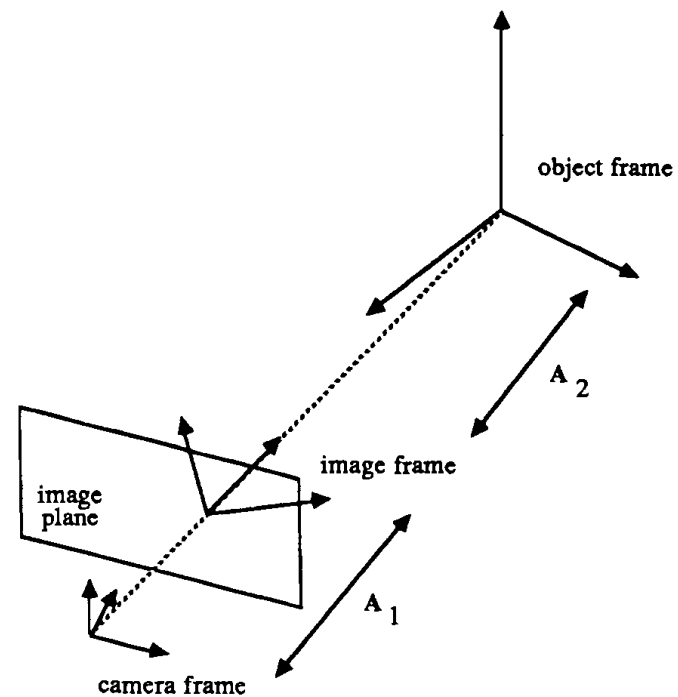

Figure 3: This figure shows the decomposition of the transformation matrix $\mathbf{A}$ into two matrices: $\mathbf{A}_{1}$ which maps the image frame into the camera frame and $\mathbf{A}_{2}$ which maps the object frame into the image frame

- We define now an image coordinate system which is rigidly attached to the projections of the object features, i.e., the image features. The projection of $L_{1}$ onto the image plane is $l_{1}$. The focal point $F$ and $l_{1}$ define a plane called the interpretation plane. Notice that this plane is rigidly attached to the camera frame since the coordinates of $l_{1}$ are measured in this frame. All the spatial interpretations of $l_{1}$, and hence $L_{1}$, belong to this plane. Let $P_{1}$ be the normal unit vector associated with this plane. Hence $P_{3}$ mentioned above is the unit vector normal to the interpretation plane associated with $l_{3}$. Let $\mathbf{J}$ be the point of intersection of the image lines $l_{1}, l_{2}$, and $l_{3}$.

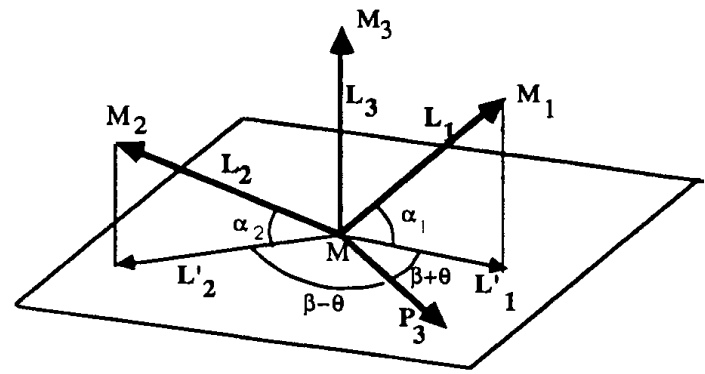

Figure 4: An "object frame" is associated with the four points. 
We are now ready to define the image coordinate system. The $\mathrm{x}$-axis lies along the line from $\mathrm{F}$ to $\mathrm{J}$. Let $k^{\prime}$ be the unit vector associated with this line. $k^{\prime}$ belongs to the three interpretation planes and hence $k^{\prime}$ is perpendicular to $P_{1}, P_{2}$, and $P_{3}$. Hence these vectors are coplanar. The image frame is defined by $k^{\prime}, P_{3}$, and their cross product, $k^{\prime} \times P_{3}$.

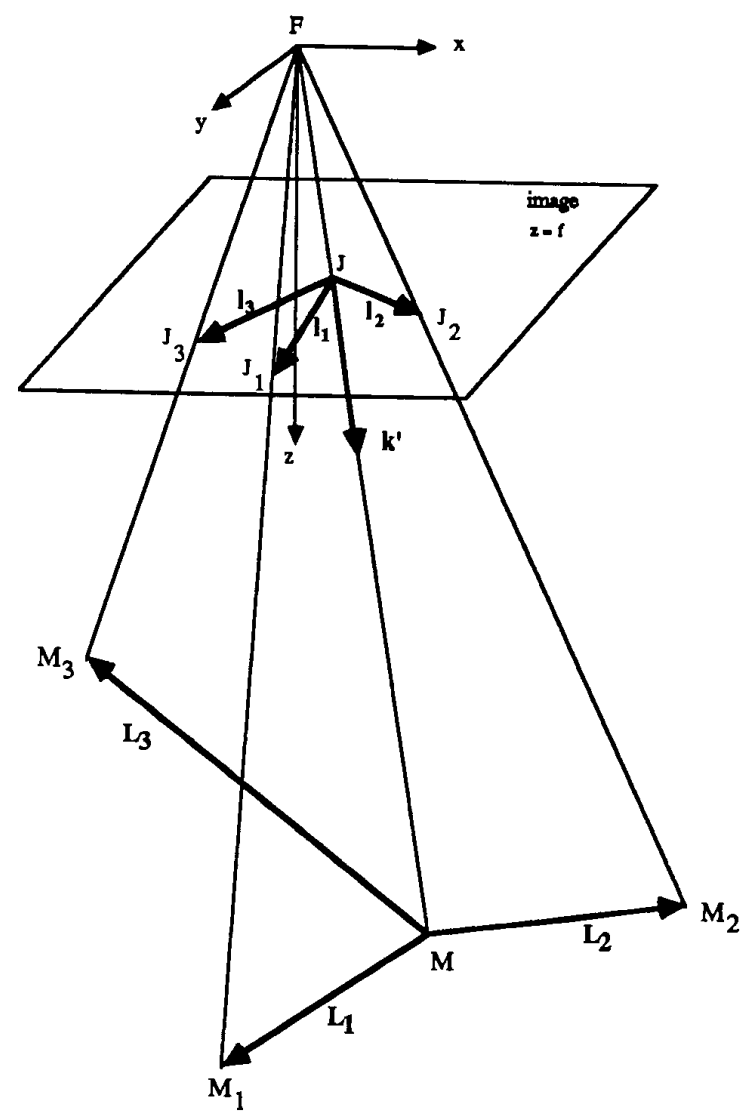

Figure 5: The geometry of the 4-point perspective problem.

A transformation between any two frames is defined by three rotations and three translations. Such a transformation may be represented by a 4 by 4 matrix (standard homogeneous coordinates). There are nine coefficients that specify the three rotations and three coefficients that specify the three translations. Next we determine the coefficients of $\mathbf{A}_{1}$ and $\mathbf{A}_{2}$.

\subsection{The matrix $\mathbf{A}_{1}$}

In order to determine the coefficients of this matrix one has to express $k^{\prime}, P_{3}$ and $k^{\prime} \times P_{3}$ in the camera frame.
We have:

$$
\begin{aligned}
k^{\prime} & =\frac{\overrightarrow{\mathrm{FJ}}}{\|\overrightarrow{\mathrm{FJ}}\|} \\
P_{3} & =\frac{l_{3} \times k^{\prime}}{\left\|l_{3} \times k^{\prime}\right\|} \\
k^{\prime} \times P_{3} & =\frac{k^{\prime} \times\left(l_{3} \times k^{\prime}\right)}{\left\|l_{3} \times k^{\prime}\right\|}=\frac{l_{3}-\left(k^{\prime} \cdot l_{3}\right) k^{\prime}}{\left\|l_{3} \times k^{\prime}\right\|}
\end{aligned}
$$

The translational parameters are given by the coordinates of $\mathbf{J}$ in the camera frame. Matrix $\mathbf{A}_{1}$ is the following:

$$
\mathbf{A}_{1}=\left(\begin{array}{cccc}
k_{x}^{\prime} & \left(P_{3}\right)_{x} & \left(k^{\prime} \times P_{3}\right)_{x} & (\overrightarrow{\mathrm{FJ}})_{x} \\
k_{y}^{\prime} & \left(P_{3}\right)_{y} & \left(k^{\prime} \times P_{3}\right)_{y} & (\overrightarrow{\mathrm{FJ}})_{y} \\
k_{z}^{\prime} & \left(P_{3}\right)_{z} & \left(k^{\prime} \times P_{3}\right)_{z} & (\overrightarrow{\mathrm{FJ}})_{z} \\
0 & 0 & 0 & 1
\end{array}\right)
$$

\subsection{The matrix $\mathbf{A}_{2}$}

We recall that $\mathbf{A}_{2}$ is the transformation matrix from the object frame (defined by $L_{3}, P_{3}$, and $L_{3} \times P_{3}$ ) to the image frame (defined by $k^{\prime}, P_{3}$, and $k^{\prime} \times P_{3}$ ). From Figure 6 which shows the interpretation plane associated with $\mathrm{F}$ and $l_{3}$ it is easy to derive an expression for $L_{3}$ :

$$
\begin{aligned}
L_{3}= & \cos \phi k^{\prime}+\sin \phi k^{\prime} \times P_{3} \\
& \text { with the constraint } 0<\phi<\pi
\end{aligned}
$$

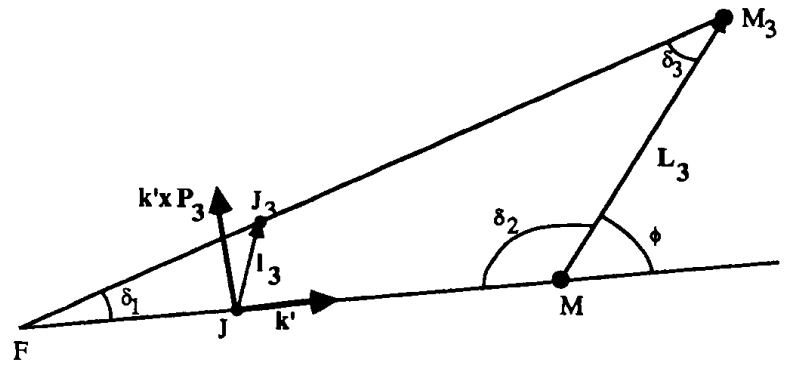

Figure 6: The interpretation plane associated with $\mathbf{F}$ and $l_{3}$

The rotational coefficients of $\mathbf{A}_{2}$ are the coordinates of $L_{3}$ in the image centered frame, i.e., eq. (6), the coordinates of $P_{3}$, i.e., 0,1 , and 0 , and the coordinates of $L_{3} \times P_{3}$. The translational coefficients are the coordinates of the vector $\mathbf{J} \vec{M}$, e.g., Figure 5. Since the direction of this last vector is the direction of $k^{\prime}$, the matrix $\mathbf{A}_{2}$ is: 


$$
\mathbf{A}_{2}=\left(\begin{array}{cccc}
\cos \phi & 0 & -\sin \phi & d_{x} \\
0 & 1 & 0 & 0 \\
\sin \phi & 0 & \cos \phi & 0 \\
0 & 0 & 0 & 1
\end{array}\right)
$$

The perspective 4-point problem is reduced now to the problem of determining values for $\theta$ (present in equations (2) and (3)), $\phi$, and $d_{x}=\|J \vec{M}\|$. Next we derive closed form expressions for these three unknowns.

\subsection{Analytic expressions for $\theta$ and $\phi$}

Two more geometric constraints are available: $L_{1}$ belongs to the interpretation plane associated with $l_{1}$. Hence $L_{1}$ is orthogonal to $P_{1}$ :

$$
L_{1} \cdot P_{1}=0
$$

Similarly we have:

$$
L_{2} \cdot P_{2}=0
$$

We express $L_{1}, L_{2}, P_{1}$, and $P_{2}$ in the image coordinate frame. By applying the transformation given by (7) to $L_{1}$ and $L_{2}$ (which are given by equations (2) and (3)) we obtain (the $\boldsymbol{k}^{\prime}$-components are not relevant for this computation):

$$
\begin{array}{r}
L_{1}=() k^{\prime}+\cos \alpha_{1} \cos (\beta+\theta) P_{3}+ \\
\left(\sin \phi \sin \alpha_{1}+\cos \phi \cos \alpha_{1} \sin (\beta+\theta)\right) k^{\prime} \times P_{3} \\
L_{2}=() k^{\prime}+\cos \alpha_{2} \cos (\beta-\theta) P_{3}+ \\
\left(\sin \phi \sin \alpha_{2}+\cos \phi \cos \alpha_{2} \sin (\beta-\theta)\right) k^{\prime} \times P_{3}
\end{array}
$$

We have already noticed that $P_{1}$ and $P_{2}$ are perpendicular to $k^{\prime}$. We have:

$$
\begin{aligned}
& P_{1}=\cos \gamma_{1} P_{3}+\sin \gamma_{1} k^{\prime} \times P_{3} \\
& P_{2}=\cos \gamma_{2} P_{3}+\sin \gamma_{2} k^{\prime} \times P_{3}
\end{aligned}
$$

Where $\gamma_{1}$ and $\gamma_{2}$ are given by (the unit vector normal to any interpretation plane, $P_{i}$ can be determined using equation (4)):

$$
\begin{aligned}
\cos \gamma_{1} & =P_{1} \cdot P_{3} \\
\sin \gamma_{1} & =\left\|P_{1} \times P_{3}\right\| \\
\cos \gamma_{2} & =P_{2} \cdot P_{3} \\
\sin \gamma_{2} & =\left\|P_{2} \times P_{3}\right\|
\end{aligned}
$$

We inject the expressions of $L_{1}, L_{2}, P_{1}$, and $P_{2}$ in equations (8) and (9) and obtain:

$$
\left(\cos \gamma_{1} \cos \beta+\sin \gamma_{1} \sin \beta \cos \phi\right) \cos \theta+
$$

$$
\begin{array}{r}
\left(-\cos \gamma_{1} \sin \beta+\sin \gamma_{1} \cos \beta \cos \phi\right) \sin \theta= \\
-\sin \gamma_{1} \sin \phi \tan \alpha_{1} \\
\left(\cos \gamma_{2} \cos \beta+\sin \gamma_{2} \sin \beta \cos \phi\right) \cos \theta+ \\
\left(\cos \gamma_{2} \sin \beta-\sin \gamma_{2} \cos \beta \cos \phi\right) \sin \theta= \\
-\sin \gamma_{2} \sin \phi \tan \alpha_{2}
\end{array}
$$

We determine $\sin \theta$ and $\cos \theta$ as a function of $\phi$ :

$$
\begin{aligned}
\cos \theta & =\frac{\sin \phi}{D}\left(-K_{1} \cos \phi+K_{2}\right) \\
\sin \theta & =\frac{\sin \phi}{D}\left(K_{3} \cos \phi+K_{4}\right)
\end{aligned}
$$

With:

$$
\begin{aligned}
& D=K_{5}-K_{6} \cos ^{2} \phi-K_{7} \cos \phi \\
& K_{1}=\sin \gamma_{1} \sin \gamma_{2} \cos \beta\left(\tan \alpha_{1}+\tan \alpha_{2}\right) \\
& K_{2}=\left(\sin \gamma_{1} \cos \gamma_{2} \tan \alpha_{1}+\cos \gamma_{1} \sin \gamma_{2} \tan \alpha_{2}\right) \sin \beta \\
& K_{3}=\sin \gamma_{1} \sin \gamma_{2} \sin \beta\left(\tan \alpha_{1}-\tan \alpha_{2}\right) \\
& K_{4}=\left(\sin \gamma_{1} \cos \gamma_{2} \tan \alpha_{1}-\cos \gamma_{1} \sin \gamma_{2} \tan \alpha_{2}\right) \cos \beta \\
& K_{5}=\cos \gamma_{1} \cos \gamma_{2} \sin 2 \beta \\
& K_{6}=\sin \gamma_{1} \sin \gamma_{2} \sin 2 \beta \\
& K_{7}=\sin \left(\gamma_{1}+\gamma_{2}\right) \cos 2 \beta
\end{aligned}
$$

Finally using the constraint $\cos ^{2} \theta+\sin ^{2} \theta=1$ we obtain:

$$
I_{1} \cos ^{4} \phi+I_{2} \cos ^{3} \phi+I_{3} \cos ^{2} \phi+I_{4} \cos \phi+I_{5}=0
$$

With:

$$
\begin{aligned}
& I_{1}=K_{1}^{2}+K_{3}^{2}+K_{6}^{2} \\
& I_{2}=2\left(K_{1} K_{2}+K_{3} K_{4}+K_{6} K_{7}\right) \\
& I_{3}=K_{2}^{2}+K_{4}^{2}-K_{1}^{2}-K_{3}^{2}+K_{7}^{2}-2 K_{5} K_{6} \\
& I_{4}=-2\left(K_{1} K_{2}+K_{3} K_{4}+K_{5} K_{7}\right) \\
& I_{5}=K_{5}^{2}-K_{2}^{2}-K_{4}^{2}
\end{aligned}
$$

The roots of equation (20) can be found in closed form or by an iterative method. For its real roots verifying the obvious constraint $|\cos \phi|<1$, we can compute $\theta$ through the formulas given by equations (18) and (19). With the values of $\phi$ and $\theta$ thus obtained we can compute $L_{1}, L_{2}$, and $L_{3}$ using equations (10), (11), and (6). The following constraint guarantees that $L_{i}$ lies in between $\mathbf{F M}$ and $\mathrm{FM}_{i}$ (Figure 6):

$$
L_{i} \cdot\left(k^{\prime} \times P_{i}\right)>0 \text { for } i=1,2,3
$$

All these constraints allow us to eliminate roots which don't correspond to an admissible geometric configuration. 


\subsection{An expression for $d_{x}$}

From Figure 6 it is easy to determine the length of $\overrightarrow{F M}$. We have:

$$
\begin{aligned}
\left\|\mathbf{M} \overrightarrow{\mathbf{M}}_{3}\right\|= & \|\dot{\mathbf{F}} \overrightarrow{\mathrm{M}}\| \cos \delta_{2}+\left\|\mathrm{F} \overrightarrow{\mathrm{M}}_{3}\right\| \cos \delta_{3} \\
& \|\overrightarrow{\mathrm{F}} \overrightarrow{\mathrm{M}}\| \sin \delta_{2}=\left\|\mathbf{F} \overrightarrow{\mathrm{M}}_{3}\right\| \sin \delta_{3}
\end{aligned}
$$

From these equations we obtain:

$$
\|\mathrm{F} \overrightarrow{\mathrm{M}}\|=\left\|\mathbf{M} \vec{M}_{3}\right\| \frac{\sin \delta_{3}}{\sin \left(\delta_{2}+\delta_{3}\right)}
$$

We also have:

$$
\begin{array}{r}
\sin \delta_{3}=\frac{\left\|\overrightarrow{\mathrm{FJ}} \overrightarrow{\mathrm{M}}_{3} \times \overrightarrow{\mathrm{M}}_{3}\right\|}{\left\|\overrightarrow{\mathrm{F}} \overrightarrow{\mathrm{J}}_{3}\right\|\left\|\mathbf{M} \overrightarrow{\mathrm{M}}_{3}\right\|} \\
\sin \left(\delta_{2}+\delta_{3}\right)=\sin \delta_{1}=\frac{\left\|\overrightarrow{\mathrm{FJ}} \times \mathbf{F} \overrightarrow{\mathrm{J}}_{3}\right\|}{\left\|\overrightarrow{\mathrm{F}} \overrightarrow{\mathrm{J}}_{3}\right\|\|\overrightarrow{\mathrm{FJ}}\|}
\end{array}
$$

Finally we obtain:

$$
\|\mathrm{F} \overrightarrow{\mathrm{M}}\|=\frac{\|\overrightarrow{\mathrm{FJ}}\|\left\|\overrightarrow{\mathrm{F}} \overrightarrow{\mathrm{J}}_{3} \times \mathrm{M}_{\mathbf{M}}\right\|}{\left\|\overrightarrow{\mathrm{FJ}} \times \overrightarrow{\mathrm{J}}_{3}\right\|}
$$

The translation vector $d_{x}$ is:

$$
d_{x}=\|\mathrm{J} \overrightarrow{\mathbf{M}}\|=\|\vec{F} \vec{M}\|-\|\overrightarrow{F J}\|
$$

\section{Special configurations}

In the previous section we derived an analytical solution for the perpsective 4-point problem in the general case. It is interesting to study some particular configurations associated with these four points.

\subsection{Four coplanar points}

In this section we show that the general formulation applies to four coplanar points. This situation corresponds to $2 \beta=(\beta+\theta)+(\beta-\theta)=\pi$, e.g., Figure 4 . In this case we obtain:

$$
\begin{aligned}
& K_{1}=K_{4}=K_{5}=K_{6}=0 \\
& I_{2}=I_{4}=0 \\
& I_{1}=K_{3}^{2} \\
& I_{3}=K_{2}^{2}-K_{3}^{2}+K_{7}^{2} \\
& I_{5}=-K_{2}^{2}
\end{aligned}
$$

The solution is given by the following equation:

$$
I_{1} \cos ^{4} \phi+I_{3} \cos ^{2} \phi+I_{5}=0
$$

It is worth noticing that the discriminant of this equation is always positive:

$$
\Delta=I_{3}^{2}-4 I_{1} I_{5}=\left(K_{2}^{2}-K_{3}^{2}+K_{7}^{2}\right)^{2}+4 K_{3}^{2} K_{2}^{2}
$$

\subsection{Three colinear image points}

Another particular situation is due to an accidental alignment: The image projections of three among the four points are colinear. Let's suppose for instance that the image points $J, J_{1}$, and $J_{3}$ are colinear. In this case the interpretation planes $P_{1}$ and $P_{3}$ are identical. Hence we have: $\cos \gamma_{1}=1$ and $\sin \gamma_{1}=0$. We obtain:

$K_{1}=K_{3}=K_{6}=0$

$I_{1}=I_{2}=0$

$I_{3}=K_{2}^{2}+K_{4}^{2}-K_{7}^{2}$

$I_{4}=-2 K_{5} K_{7}$

$I_{5}=K_{5}^{2}-K_{2}^{2}-K_{4}^{2}$

The solution is given by:

$$
I_{3} \cos ^{2} \phi+I_{4} \cos \phi+I_{5}=0
$$

Real roots exist for this equation if and only if its discriminant is positive:

$\Delta=\sin ^{2} \gamma_{2}\left(\tan ^{2} \alpha_{2}-\cos ^{2} 2 \beta\right)-\cos ^{2} \gamma_{2} \geq 0$

The accidental alignment described here corresponds to a 3-line spatial vertex being projected onto the image as a T-junction. The result of this section is that such a $\mathrm{T}$-junction may have a three-dimensional interpretation.

\subsection{A right vertex}

Another particular case occurs when the four points form a right vertex, i.e., $L_{1}, L_{2}$, and $L_{3}$ are mutually orthogonal:

$$
L_{i} \cdot L_{j}=0 \text { for } i \neq j
$$

In this case a simpler solution than the general case can be derived. Notice first that equation (6) can also be written for $L_{1}$ and $L_{2}$ :

$$
L_{i}=\cos \phi_{i} k^{\prime}+\sin \phi_{i} k^{\prime} \times P_{3} \text { for } i=1,2,3
$$

For notation homogeneity $\phi$ is replaced by $\phi_{3}$. We obtain:

$L_{i} \cdot L_{j}=\cos \phi_{i} \cos \phi_{j}+\sin \phi_{i} \sin \phi_{j}\left(P_{i} \cdot P_{j}\right)$ with $i \neq j$

We have already mentioned that $P_{1}, P_{2}$, and $P_{3}$ are coplanar (they are all orthogonal to $k^{\prime}$ ). Hence, the three dot products $P_{i} \cdot P_{j}$ cannot be simultaneously null. There are three possible situations:

1. $P_{1} \cdot P_{2}=0, P_{1} \cdot P_{3} \neq 0$, and $P_{2} \cdot P_{3} \neq 0$. We obtain $\phi_{1}=\phi_{2}=0$ which is impossible because it corresponds to two space points which project onto the image at the same location, e.g., Figure 6; 
2. $P_{1} \cdot P_{2}=0, P_{1} \cdot P_{3}=0$, and $P_{2} \cdot P_{3} \neq 0 . P_{1}$ is perpendicular to both $P_{2}$ and $P_{3}$, hence they are colinear, $\left|P_{2} \cdot P_{3}\right|=1$. We obtain:

$$
\begin{aligned}
& \cos \phi_{1} \cos \phi_{2}=0 \\
& \cos \phi_{1} \cos \phi_{3}=0
\end{aligned}
$$

$\sin \phi_{2} \sin \phi_{3} \pm \cos \phi_{2} \cos \phi_{3}=0$

Which gives the solutions: $\phi_{1}=\pi / 2 \phi_{2} \pm \phi_{3}=\pi / 2$, and

3. $P_{1} \cdot P_{2} \neq 0, P_{1} \cdot P_{3} \neq 0$, and $P_{2} \cdot P_{3} \neq 0$. We obtain:

$$
\begin{array}{r}
\tan \phi_{2}=\tan \phi_{3} \frac{P_{1} \cdot P_{3}}{P_{1} \cdot P_{2}} \\
\tan \phi_{1}=\tan \phi_{3} \frac{P_{2} \cdot P_{3}}{P_{1} \cdot P_{2}} \\
\tan ^{2} \phi_{3}=-\frac{P_{1} \cdot P_{2}}{\left(P_{1} \cdot P_{3}\right)\left(P_{2} \cdot P_{3}\right)}
\end{array}
$$

In this case solutions exist under the constraint: $\left(P_{1} \cdot P_{2}\right)\left(P_{1} \cdot P_{3}\right)\left(P_{2} \cdot P_{3}\right)<0$.

This last constraint can be used to select image junctions that have a right vertex interpretation. Figure 7 shows the image of four objects. Figure 8 shows the lines extracted from the above image (top) and the 3line junctions having a right vertex interpretation.

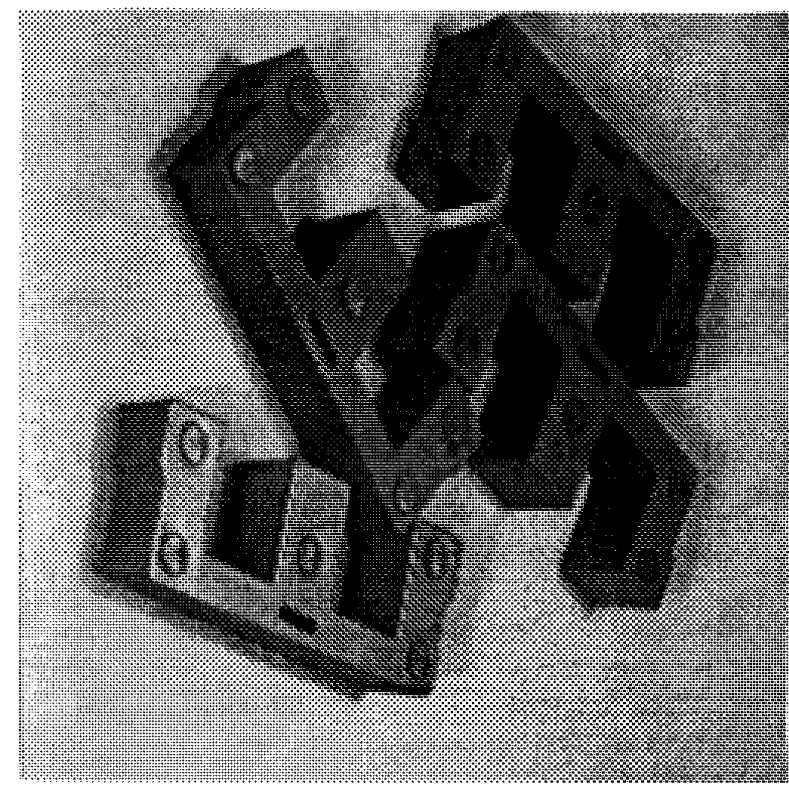

Figure 7: An image of a few objects

\section{Discussion}

In this paper we derived an analytic solution for computing the exterior camera parameters from four correspondence points in general positions. This solution is of the same complexity as for three points and is particularly simple for such configurations as four coplanar points or four points forming a right vertex. Such an analytic formulation allows fast numerical computation which is desirable in many applications such as on line calibration (hand/eye coordination, navigation) and/or object recognition and positioning from a single view.

\section{References}

[1] R.A. Brooks, A.M. Flynn, and Th. Marill. Self Calibration of Motion and Stereo Vision for Mobile Robots. In The Fourth International Symposium of Robotics Research, pages 277-286, MIT Press, Santa Cruz, CA, August 1987.

[2] M. Dhome, M. Richetin, J.T. Lapreste, and G. Rives. Determination of the Attitude of $3 \mathrm{D} \mathrm{Ob}$ jects from a Single Perspective View. IEEE Transactions on Pattern Analysis and Machine Intelligence, 1988. To appear.

[3] O. D. Faugeras and G. Toscani. The Calibration Problem for Stereo. In Proc. Computer Vision and Pattern Recognition, pages 15-20, Miami Beach, Florida, USA, June 1986.

[4] M.A. Fischler and R.C. Bolles. Random Sample Consensus: A Paradigm for Model Fitting with Applications to Image Analysis and $\mathrm{Au}-$ tomated Cartography. Communications of the $A C M, 24(6): 381-395$, June 1981.

[5] W.E.L. Grimson and T. Lozano-Perez. Modelbased Recognition and Localization from Sparse Range or Tactile Data. International Journal of Robotics Research, 3(3):3-35, Fall 1984.

[6] R. Horaud. New Methods for Matching 3-D Objects with Single Perspective Views. IEEE Trans. on Pattern Analysis and Machine Intelligence, PAMI-9(3):401-412, May 1987.

[7] D.P. Huttenlocher and S. Ullman. Object Recognition Using Alignment. In The First International Conference on Computer Vision, pages 102111, London, England, June 1987.

[8] Y. Lamdan, J.T. Schwartz, and H.J. Wolfson. On the Recocgnition fo 3D Objects from 2D Images. 
In IEEE International Conference on Robotics and Automation, pages 1407-1413, Philadelphia, Penn, USA, April 1988.

[9] D. Lowe. Three-dimensional Object Recognition from Single Two-dimensional Images. Artificial Intelligence, 31:355-395, 1987.

[10] P. Rives, P. Bouthémy, B. Prasada, and E Dubois. Recovering the Orientation and the Position of a Rigid Body in Space from a Single View. Technical Report, INRS-Télécommunications, 3, place du Commerce, Ile-des-Soeurs, Verdun, H3E 1H6, Québec, Canada, 1981.

[11] T. Shakunaga and H. Kaneko. Shape from Angles under Perspective Projection. In Proc. IEEE International Conference on Computer Vision, pages 671-678, Tampa, Florida, USA, December 1988.

[12] D.W. Thompson and J.L. Mundy. Threedimensional Model Matching from an Unconstrained Viewpoint. In IEEE International Conference on Robotics and Automation, pages 208220, Raleigh, North Carolina, USA, March 1987.

[13] R. Y. Tsai. A Versatile Camera Calibration Technique for High-Accuracy 3D Machine Vision Metrology Using Off-the-Shelf TV Cameras and Lenses. IEEE Journal of Robotics and Automation, RA-3(4):323-344, August 1987.

[14] R.Y. Tsai and R.K. Lenz. Real Time Versatile Robotics Hand/Eye Calibration using 3D Machine Vision. In IEEE International Conference on Robotics and Automation, pages 554-461, Philadelphia, Penn, USA, April 1988.
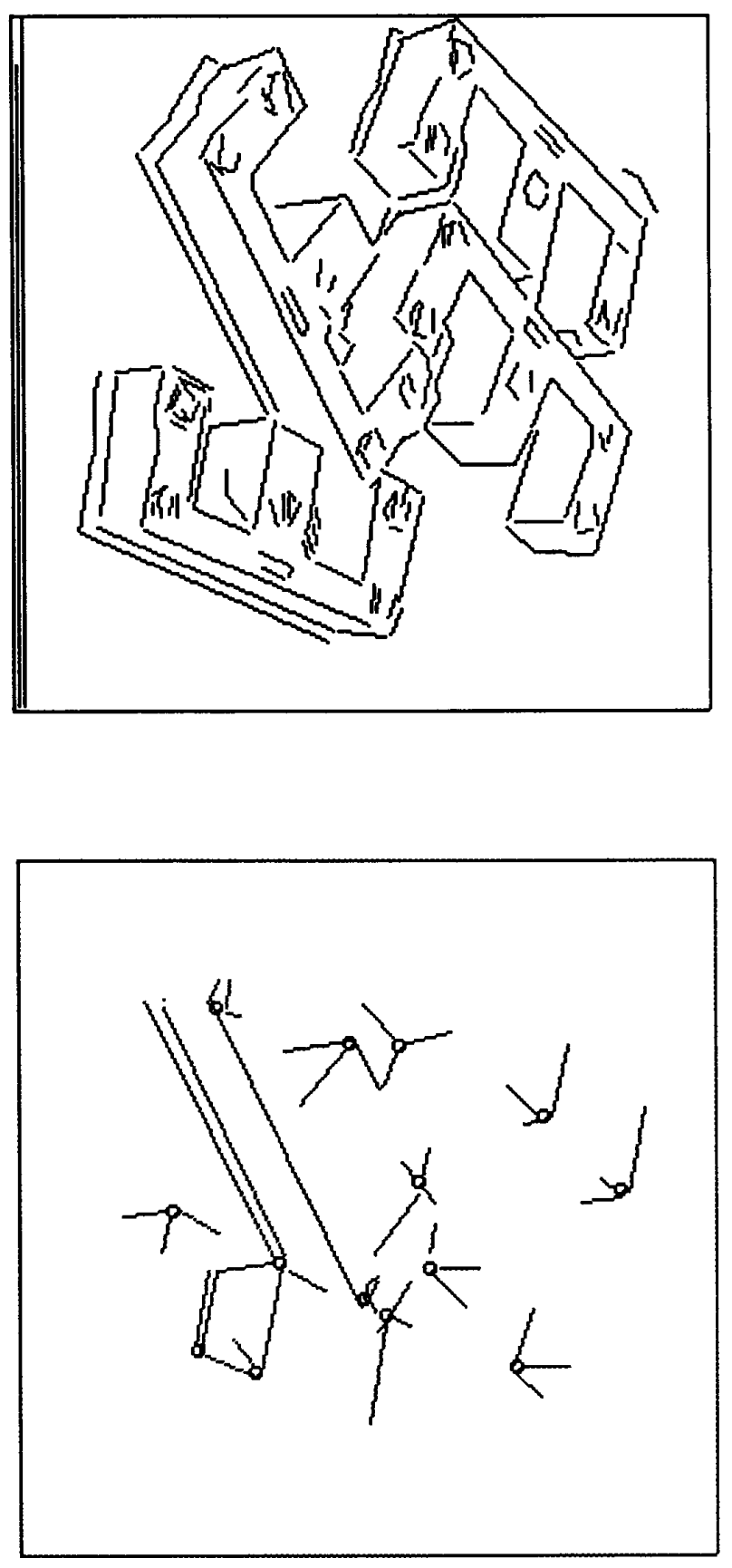

Figure 8: The lines extracted from the image and the set of 3 -line junctions that have a right vertex interpretation 